\title{
ON A SINGULAR SUBLINEAR POLYHARMONIC PROBLEM
}

\author{
SONIA BEN OTHMAN
}

Received 27 June 2005; Accepted 5 September 2005

This paper deals with a class of singular nonlinear polyharmonic equations on the unit ball $B$ in $\mathbb{R}^{n}(n \geq 2)$ where the combined effects of a singular and a sublinear term allow us by using the Schauder fixed point theorem to establish an existence result for the following problem: $(-\Delta)^{m} u=\varphi(\cdot, u)+\psi(\cdot, u)$ in $B$ (in the sense of distributions), $u>0$, $\lim _{|x| \rightarrow 1} u(x) /(1-|x|)^{m-1}=0$. Our approach is based on estimates for the polyharmonic Green function on $B$ with zero Dirichlet boundary conditions.

Copyright (C) 2006 Sonia Ben Othman. This is an open access article distributed under the Creative Commons Attribution License, which permits unrestricted use, distribution, and reproduction in any medium, provided the original work is properly cited.

\section{Introduction}

In this paper, we study the existence and the asymptotic behaviour of a positive solution for the following higher-order singular problem involving sublinear term

$$
\begin{gathered}
(-\Delta)^{m} u=\varphi(\cdot, u)+\psi(\cdot, u) \text { in } B \quad(\text { in the sense of distributions }), \\
u>0 \\
\lim _{|x| \rightarrow 1} \frac{u(x)}{(1-|x|)^{m-1}}=0
\end{gathered}
$$

where $B$ is the unit ball in $\mathbb{R}^{n}(n \geq 2)$ and $m$ is a positive integer.

Our motivation to study the problem $(P)$ comes from [11], where Shi and Yao investigated the existence of nonnegative solutions for the following elliptic problem:

$$
\begin{gathered}
\Delta u+k(x) u^{-\gamma}+\lambda u^{\alpha}=0 \quad \text { in } \Omega, \\
u(x)>0, \quad x \in \Omega,
\end{gathered}
$$

where $\Omega$ is a bounded $C^{1,1}$ domain in $\mathbb{R}^{n}(n \geq 2), \gamma, \alpha$ are two constants in $(0,1), \lambda$ is 
2 Existence result for a nonlinear polyharmonic problem

a real parameter, and $k$ is a Hölder continuous function in $\bar{\Omega}$. The pure singular elliptic equation

$$
\Delta u+p(x) u^{-\gamma}=0, \quad \gamma>0, x \in D \subseteq \mathbb{R}^{n},
$$

has been extensively studied for both bounded and unbounded domain $D$ in $\mathbb{R}^{n}(n \geq 2)$. We refer to ([3-5, 7-9], and references therein) for various existence and uniqueness results related to solutions for above equation. On the other hand, the problem $(P)$ with a sublinear term $\psi(\cdot, u)$ and a singular term $\varphi(\cdot, u)=0$, has been studied in the unit ball by Mâagli et al. in [10]. Thus a natural question to ask is, for more general singular and sublinear terms combined in the nonlinearity, whether or not the problem $(P)$ has a solution, which we aim to study in this paper.

Our tools are based essentially on some inequalities satisfied by the Green function $G_{m, n}$ of $(-\Delta)^{m}$ in $B$ with Dirichlet boundary conditions $(\partial / \partial \nu)^{j} u=0 ; 0 \leq j \leq m-1$. This allows us to state some properties of functions in the Kato class $K_{m, n}(B)=K_{m, n}$ which was introduced in [1] (see Definition 1.1 below).

In [2], Boggio showed that $G_{m, n}$ is positive and it is given by

$$
G_{m, n}(x, y)=k_{m, n}|x-y|^{2 m-n} \int_{1}^{[x, y] /|x-y|} \frac{\left(v^{2}-1\right)^{m-1}}{v^{n-1}} d v,
$$

where $k_{m, n}$ is a positive constant and $[x, y]^{2}=|x-y|^{2}+\left(1-|x|^{2}\right)\left(1-|y|^{2}\right)$ for $x, y \in B$. Note that the positivity does not hold for the Green function of the $m$-polyharmonic operator in arbitrary bounded domain (see, e.g., [6]).

Only for $m=1$, we do not have this restriction.

Definition 1.1. A Borel measurable function $q$ in $B$ belongs to the class $K_{m, n}$ if $q$ satisfies the following condition:

$$
\lim _{\alpha \rightarrow 0}\left(\sup _{x \in B} \int_{B \cap B(x, \alpha)}\left(\frac{\delta(y)}{\delta(x)}\right)^{m} G_{m, n}(x, y)|q(y)| d y\right)=0,
$$

where $\delta(x)=1-|x|$ is the Euclidian distance between $x$ and $\partial B$.

We refer in this paper to the potential of a measurable nonnegative function $f$, defined on $B$ by

$$
V f(x)=\int_{B} G_{m, n}(x, y) f(y) d y .
$$

Our plan in this paper is as follows. Section 2 is devoted to collect some preliminary results about the Green function $G_{m, n}(x, y)$ and the class $K_{m, n}$. In Section 3, we suppose that $\varphi$ and $\psi$ satisfy the following hypotheses.

$\left(\mathrm{H}_{1}\right) \varphi$ is a nonnegative Borel measurable function on $B \times(0, \infty)$, continuous and nonincreasing with respect to the second variable.

$\left(\mathrm{H}_{2}\right)$ For all $c>0, x \mapsto \varphi\left(x, c(\delta(x))^{m}\right) /(\delta(x))^{m-1} \in K_{m, n}$.

$\left(\mathrm{H}_{3}\right) \psi$ is a nonnegative Borel measurable function on $B \times(0, \infty)$, continuous with respect to the second variable such that there exist a nontrivial nonnegative function $p$ 
and a nonnegative function $q$ on $B$ satisfying for $x \in B$ and $t>0$,

$$
\begin{gathered}
x \longmapsto \frac{q(x)}{(\delta(x))^{m-1}} \in K_{m, n}, \\
p(x) f(t) \leq \psi(x, t) \leq q(x) g(t) \quad \text { for }(x, t) \in B \times(0, \infty),
\end{gathered}
$$

where $f$ is a measurable nondecreasing function on $[0, \infty)$ satisfying

$$
\lim _{t \rightarrow 0^{+}} \frac{f(t)}{t}=+\infty
$$

and $g$ is a nonnegative measurable function locally bounded on $[0, \infty)$ satisfying

$$
\limsup _{t \rightarrow \infty} \frac{g(t)}{t}<\|V q\|_{\infty}
$$

Using a fixed point argument, we will state the following existence result.

Theorem 1.2. Under hypotheses $\left(H_{1}\right)-\left(H_{3}\right)$, problem $(P)$ has a positive continuous solution $u$ satisfying for each $x \in B$,

$$
a(\delta(x))^{m} \leq u(x) \leq V\left(\varphi\left(\cdot, a \delta(\cdot)^{m}\right)\right)(x)+b V q(x),
$$

where $a, b$ are positive constants.

Typical examples of nonlinearities satisfying $\left(\mathrm{H}_{1}\right)-\left(\mathrm{H}_{3}\right)$ are

$$
\varphi(x, t)=p(x)(\delta(x))^{m \gamma+m-1} t^{-\gamma}
$$

for $\gamma \geq 0$, and

$$
\psi(x, t)=q(x)(\delta(x))^{m-1} t^{\alpha} \log \left(1+t^{\beta}\right)
$$

for $\alpha, \beta \geq 0$ such that $\alpha+\beta<1$, where $p$ and $q$ are nonnegative functions in $K_{m, n}$.

Notations. In order to simplify our statements, we define some convenient notation.

(i) $B:=\left\{x \in \mathbb{R}^{n} ;|x|<1\right\}$ with $n \geq 2$.

(ii) We denote $s \wedge t=\min (s, t)$ and $s \vee t=\max (s, t)$ for $s, t \in \mathbb{R}$.

(iii) For $(x, y) \in B^{2},[x, y]^{2}=|x-y|^{2}+\left(1-|x|^{2}\right)\left(1-|y|^{2}\right)$.

(iv) $\mathscr{B}(B)$ denotes the set of Borel measurable functions in $B$ and $\mathscr{B}^{+}(B)$ is the set of nonnegative ones.

(v) $C_{0}(B):=\left\{\omega\right.$ continuous on $B$ and $\left.\lim _{|x| \rightarrow 1} \omega(x)=0\right\}$. We recall that $C_{0}(B)$ is a Banach space with the uniform norm $\|\omega\|_{\infty}=\sup _{x \in B}|\omega(x)|$.

(vi) Let $f$ and $g$ be two positive functions on a set $S$. We call, $f \sim g$, if there is $c>0$ such that

$$
\frac{1}{c} g(x) \leq f(x) \leq c g(x) \quad \forall x \in S
$$


4 Existence result for a nonlinear polyharmonic problem

$f \preceq g$, if there is $c>0$ such that

$$
f(x) \leq \operatorname{cg}(x) \quad \forall x \in S \text {. }
$$

(vii) For any $f \in \mathscr{B}(B)$, we put

$$
\|\varphi\|_{B}:=\sup _{x \in B} \int_{B}\left(\frac{\delta(y)}{\delta(x)}\right)^{m} G_{m, n}(x, y)|f(y)| d y .
$$

\section{Properties of the Green function and the Kato class $K_{m, n}$}

The existence result that we are going to prove suggests to collect in this section some estimates on the Green function and some properties of functions belonging to the Kato class $K_{m, n}$.

Proposition 2.1. For each $m \geq 1$, the following estimates are satisfied on $B^{2}$ :

$$
\begin{gathered}
G_{m, n}(x, y) \preceq(\delta(x) \delta(y))^{m-1} G_{1, n}(x, y), \\
(\delta(x) \delta(y))^{m} \preceq G_{m, n}(x, y) .
\end{gathered}
$$

For $n>2 m$,

$$
G_{m, n}(x, y) \preceq \frac{1}{|x-y|^{n-2 m}}
$$

For $n<2 m$,

$$
G_{m, n}(x, y) \preceq \frac{(\delta(x) \delta(y))^{m}}{[x, y]^{n}}
$$

For $n=2 m$,

$$
G_{m, n}(x, y) \preceq \frac{(\delta(x))^{m-1}(\delta(y))^{m-1}}{[x, y]^{2(m-1)}} \log \left(1+\frac{\delta(x) \delta(y)}{|x-y|^{2}}\right) .
$$

Proof. The proof of assertions (2.1)-(2.4) can be found in [1]. If $n=2 m$, it follows from (1.3) that for $(x, y)$ in $B^{2}$, we have

$$
\begin{aligned}
G_{m, n}(x, y) & \preceq \int_{1}^{[x, y] /|x-y|} \frac{1}{v}\left(1-\frac{1}{v^{2}}\right)^{m-1} d v \\
& \preceq\left(1-\frac{|x-y|^{2}}{[x, y]^{2}}\right)^{m-1} \log \left(\frac{[x, y]}{|x-y|}\right) \\
& \preceq \frac{(\delta(x))^{m-1}(\delta(y))^{m-1}}{[x, y]^{2(m-1)}} \log \left(1+\frac{\delta(x) \delta(y)}{|x-y|^{2}}\right),
\end{aligned}
$$

which gives (2.5). 
Proposition 2.2 (see [10]). Let $q$ be a nonnegative function in $K_{m, n}$. Then the following assertions hold:

(i) $\|q\|_{B}<\infty$,

(ii) the function $x \mapsto(\delta(x))^{2 m-1} q \in L^{1}(B)$. In the sequel, we will give some estimates on the radial potential

$$
x \longmapsto \int_{S^{n-1}} G_{m, n}(x, r \omega) d \sigma(\omega)
$$

where $\sigma$ is the normalized measure on the unit sphere $S^{n-1}$ of $\mathbb{R}^{n}$.

Proposition 2.3. For $x \in B$, and $r \in(0,1)$,

$$
\int_{S^{n-1}} G_{m, n}(x, r \omega) d \sigma(\omega) \preceq(\delta(x))^{m-1}(1-r)^{m-1} \rho(|x| \vee r),
$$

where for $t \in(0,1)$,

$$
\rho(t)= \begin{cases}\frac{1-t}{t^{(n-2 m)^{+}}} & \text {if } n \neq 2 m, \\ \log \frac{1}{t} & \text { if } n=2 m,\end{cases}
$$

and $(n-2 m)^{+}=\max (0, n-2 m)$.

Proof. To show the claim, we use inequalities in Proposition 2.1.

For $x \in B$ and $r \in(0,1)$, we distinguish three cases.

(i) If $n>2 m$, we obtain by (2.1) and elementary calculus that

$$
\begin{aligned}
\int_{S^{n-1}} G_{m, n}(x, r \omega) d \sigma(\omega) & \preceq(\delta(x))^{m-1}(1-r)^{m-1} \int_{S^{n-1}} G_{1, n}(x, r \omega) d \sigma(\omega) \\
& \preceq(\delta(x))^{m-1}(1-r)^{m-1} \frac{1-|x| \vee r}{(|x| \vee r)^{n-2}}
\end{aligned}
$$

On the other hand, by (2.3) we have

$$
\begin{aligned}
\int_{S^{n-1}} G_{m, n}(x, r \omega) d \sigma(\omega) & \preceq \int_{S^{n-1}} \frac{d \sigma(\omega)}{|x-r \omega|^{n-2 m}} \\
& \preceq \int_{0}^{\pi} \frac{(\sin \theta)^{n-2}}{\left(|x|^{2}+r^{2}-2 r|x| \cos \theta\right)^{(n-2 m) / 2}} d \theta \\
& \preceq \int_{0}^{\pi} \frac{(\sin \theta)^{n-2}}{\left[((|x| \wedge r)-(|x| \vee r) \cos \theta)^{2}+(|x| \vee r)^{2}(\sin \theta)^{2}\right]^{(n-2 m) / 2}} d \theta \\
& \preceq \frac{1}{(|x| \vee r)^{n-2 m}} .
\end{aligned}
$$


6 Existence result for a nonlinear polyharmonic problem

Using the fact that for each $a, b>0$, we have $\min (a, b) \sim a b /(a+b)$, we obtain

$$
\begin{aligned}
\int_{S^{n-1}} & G_{m, n}(x, r \omega) d \sigma(\omega) \\
& \preceq \min \left(\frac{1}{(|x| \vee r)^{n-2 m}},(\delta(x))^{m-1}(1-r)^{m-1} \frac{1-|x| \vee r}{(|x| \vee r)^{(n-2)}}\right) \\
& \preceq \frac{(1-|x| \vee r)^{m}(1-|x| \wedge r)^{m-1}}{(|x| \vee r)^{n-2 m}}\left(\frac{1}{(|x| \vee r)^{2 m-1}+(1-|x| \vee r)^{2 m-1}}\right) \\
& \preceq \frac{(1-|x| \vee r)^{m}(1-|x| \wedge r)^{m-1}}{(|x| \vee r)^{n-2 m}} .
\end{aligned}
$$

(ii) If $n=2 m$, then we have from (2.5)

$$
\begin{aligned}
& \int_{S^{n-1}} G_{m, n}(x, r \omega) d \sigma(\omega) \\
& \preceq(\delta(x))^{m-1}(1-r)^{m-1} \int_{0}^{\pi} \frac{(\sin \theta)^{2 m-2}}{\left(|x|^{2} r^{2}+1-2 r|x| \cos \theta\right)^{m-1}} \\
& \times \log \left(1+\frac{\left(1-|x|^{2}\right)\left(1-r^{2}\right)}{\left(|x|^{2}+r^{2}-2 r|x| \cos \theta\right)}\right) d \theta \\
& \preceq(\delta(x))^{m-1}(1-r)^{m-1} \int_{0}^{\pi} \frac{(\sin \theta)^{2(m-1)}}{\left((|x| r-\cos \theta)^{2}+(\sin \theta)^{2}\right)^{m-1}} \\
& \times \log \left(1+\frac{\left(1-|x|^{2}\right)\left(1-r^{2}\right)}{\left(|x|^{2}+r^{2}-2 r|x| \cos \theta\right)}\right) d \theta \\
& \preceq(\delta(x))^{m-1}(1-r)^{m-1} \int_{0}^{\pi} \log \left(1+\frac{\left(1-|x|^{2}\right)\left(1-r^{2}\right)}{\left(|x|^{2}+r^{2}-2 r|x| \cos \theta\right)}\right) d \theta \\
& \preceq(\delta(x))^{m-1}(1-r)^{m-1} \log \left(\frac{1}{|x| \vee r}\right) .
\end{aligned}
$$

(iii) If $n<2 m$, then applying (2.4) we have by elementary calculus

$$
\begin{aligned}
& \int_{S^{n-1}} G_{m, n}(x, r \omega) d \sigma(\omega) \\
& \quad \preceq(\delta(x))^{m}(1-r)^{m} \int_{0}^{\pi} \frac{(\sin \theta)^{n-2}}{\left(|x|^{2} r^{2}+1-2 r|x| \cos \theta\right)^{n / 2}} \\
& \quad \preceq(\delta(x))^{m}(1-r)^{m} \int_{0}^{\pi} \frac{(\sin \theta)^{n-2}}{\left((|x| r-\cos \theta)^{2}+(\sin \theta)^{2}\right)^{(n-2) / 2}} \frac{1}{\left(|x|^{2} r^{2}+1-2 r|x| \cos \theta\right)} d \theta
\end{aligned}
$$




$$
\begin{aligned}
& \preceq(\delta(x))^{m}(1-r)^{m} \int_{0}^{\pi} \frac{1}{\left(|x|^{2} r^{2}+1-2 r|x| \cos \theta\right)} d \theta \\
& \preceq(\delta(x))^{m}(1-r)^{m} \frac{1}{1-r|x|} \preceq(1-|x| \vee r)^{m}(1-|x| \wedge r)^{m-1} .
\end{aligned}
$$

This completes the proof.

We characterize in the following the radial functions in the class $K_{m, n}$.

Proposition 2.4. Let a be a radial function in $B$, then the following assertions are equivalent:

(1) $a \in K_{m, n}$,

(2) $\int_{0}^{1} r^{n-1}(1-r)^{2(m-1)} \rho(r)|a(r)| d r<\infty$,

where $\rho$ is the function defined by (2.9).

Proof. See [1, Proposition 3.8].

Example 2.5. Let $q$ be the function defined in $B$ by

$$
q(x)=\frac{1}{(\delta(x))^{\lambda}(\log 2 / \delta(x))^{\mu}} .
$$

By Proposition 2.4, $q \in K_{m, n}$ if and only if $\lambda<2 m$ and $\mu \in \mathbb{R}$ or $\lambda=2 m$ and $\mu>1$.

Example 2.6. Let $p>\max (n / 2 m, 1)$, let $q \geq 1$ such that $1 / p+1 / q=1$, and let $a$ be a radial function in $L^{p}(B)$. Then for $\lambda<2 m-1 / p$ and $\mu \in \mathbb{R}$ or $\lambda=2 m-1 / p$ and $\mu>1 / q$, the function

$$
x \longmapsto \frac{a(x)}{\delta(x)^{\lambda}(\log 2 / \delta(x))^{\mu}}
$$

is in $K_{m, n}$.

Indeed, using the Hölder inequality we obtain

$$
\begin{gathered}
\int_{0}^{1} r^{n-1} \frac{(1-r)^{2(m-1)-\lambda}}{(\log (2 /(1-r)))^{\mu}} \rho(r)|a(r)| d r \\
\leq\|a\|_{p}\left(\int_{0}^{1} r^{n-1}(\rho(r))^{q} \frac{(1-r)^{[2(m-1)-\lambda] q}}{(\log (2 /(1-r)))^{\mu q}} d r\right)^{1 / q} \\
=\|a\|_{p}\left[\left(\int_{0}^{1 / 2} r^{n-1}(\rho(r))^{q} \frac{(1-r)^{[2(m-1)-\lambda] q}}{(\log (2 /(1-r)))^{\mu q}} d r\right)^{1 / q}\right. \\
\left.\quad+\left(\int_{1 / 2}^{1} r^{n-1}(\rho(r))^{q} \frac{(1-r)^{[2(m-1)-\lambda] q}}{(\log (2 /(1-r)))^{\mu q}} d r\right)^{1 / q}\right] \\
=\|a\|_{p}\left(I_{1}+I_{2}\right) .
\end{gathered}
$$

Since for $r \in(0,1 / 2]$, we have $1-r \sim 1$, then we deduce from (2.9) that $I_{1}<\infty$. 
8 Existence result for a nonlinear polyharmonic problem

On the other hand, using that $\log (1 / r) \sim 1-r$, for $r \in[1 / 2,1)$, then we obtain by (2.9),

$$
I_{2} \preceq\left(\int_{4}^{\infty} \frac{d r}{t^{(2 m-\lambda-1) q+2}(\log t)^{\mu q}}\right)^{1 / q} .
$$

Hence, we have obviously $I_{2}<\infty$, for $\lambda<2 m-1 / p$ and $\mu \in \mathbb{R}$ or $\lambda=2 m-1 / p$ and $\mu>$ $1 / q$. So, the result follows from Proposition 2.4 .

Remark 2.7. For $x \in B$, put $\varphi_{\lambda, \mu}(x)=1 / \delta(x)^{\lambda}(\log (2 / \delta(x)))^{\mu}$. Then it follows from (2.2) that there exists a constant $c>0$ such that for each $a \in \mathscr{B}^{+}(B)$,

$$
c \delta(x)^{m} \int_{B}(\delta(y))^{m} a(y) \varphi_{\lambda, \mu}(x) d y \leq V\left(a \varphi_{\lambda, \mu}\right)(x) .
$$

In the next proposition, we will give upper estimates on $V\left(a \varphi_{\lambda, \mu}\right)$.

Proposition 2.8. Let $p>\max (n / 2 m, 1)$ and let $q \geq 1$ such that $1 / p+1 / q=1$. Then for $\lambda<m+1-1 / p$ and $\mu \in \mathbb{R}$ or for $\lambda=m+1-1 / p$ and $\mu>1 / q$, there exists a constant $c>0$, such that for each nonnegative radial function $a \in L^{p}(B)$ and $x \in B$, the following estimates hold:

$$
V\left(a \varphi_{\lambda, \mu}\right)(x) \leq c\|a\|_{p} \times \begin{cases}(\delta(x))^{2 m-\lambda-(1 / p)}\left(\log \frac{2}{\delta(x)}\right)^{-\mu} & \text { if } m-\frac{1}{p}<\lambda<m+1-\frac{1}{p}, \mu \in \mathbb{R}, \\ (\delta(x))^{m} & \text { if } \lambda<m-\frac{1}{p}, \mu \in \mathbb{R}, \\ (\delta(x))^{m}\left(\log \log \frac{4}{\delta(x)}\right)^{1 / q} & \text { if } \lambda=m-\frac{1}{p}, \mu=\frac{1}{q}, \\ (\delta(x))^{m}\left(\log \frac{2}{\delta(x)}\right)^{1 / q-\mu} & \text { if } \lambda=m-\frac{1}{p}, \mu<\frac{1}{q}, \\ (\delta(x))^{m} & \text { if } \lambda=m-\frac{1}{p}, \mu>\frac{1}{q}, \\ (\delta(x))^{m-1}\left(\log \frac{2}{\delta(x)}\right)^{1 / q-\mu} & \text { if } \lambda=m+1-\frac{1}{p}, \mu>\frac{1}{q} .\end{cases}
$$

Proof. Let $p>\max (n / 2 m, 1)$, let $q \geq 1$ such that $1 / p+1 / q=1$ and let $a(x)=a(|x|)$ be a nonnegative function in $L^{p}(B)$. Using Proposition 2.3 and the Hölder inequality, we 
obtain that for each $x \in B$,

$$
\begin{aligned}
V\left(a \varphi_{\lambda, \mu}\right)(x) & \leq c \int_{0}^{1} r^{n-1} \frac{a(r)}{(1-r)^{\lambda}(\log (2 /(1-r)))^{\mu}}\left(\int_{S^{n-1}} G_{m, n}(x, r \omega) d \sigma(\omega)\right) d r \\
& \leq c(\delta(x))^{m-1} \int_{0}^{1} r^{n-1} \frac{(1-r)^{m-\lambda-1}}{(\log (2 /(1-r)))^{\mu}} \rho(|x| \vee r) a(r) d r \\
& \leq c(\delta(x))^{m-1}\|a\|_{p}\left(\int_{0}^{1} r^{n-1} \frac{(1-r)^{(m-\lambda-1) q}}{(\log (2 /(1-r)))^{\mu q}}(\rho(|x| \vee r))^{q} d r\right)^{1 / q} .
\end{aligned}
$$

First, if $|x| \in(0,1 / 2]$, then we deduce by (2.9) that

$$
\begin{aligned}
& \left(\int_{0}^{1} r^{n-1} \frac{(1-r)^{(m-\lambda-1) q}}{(\log (2 /(1-r)))^{\mu q}}(\rho(|x| \vee r))^{q} d r\right)^{1 / q} \\
& \quad \preceq\left(\int_{0}^{1} r^{n-1} \frac{(1-r)^{(m-\lambda-1) q}}{(\log (2 /(1-r)))^{\mu q}}(\rho(r))^{q} d r\right)^{1 / q} \preceq 1 .
\end{aligned}
$$

Now, if $|x| \in[1 / 2,1)$, we have $\rho(x) \preceq(1-|x|)$ and

$$
\begin{aligned}
& \left(\int_{0}^{1} r^{n-1} \frac{(1-r)^{(m-\lambda-1) q}}{(\log (2 /(1-r)))^{\mu q}}(\rho(|x| \vee r))^{q} d r\right)^{1 / q} \\
& \quad \preceq \delta(x)\left(\int_{0}^{|x|} r^{n-1} \frac{(1-r)^{(m-\lambda-1) q}}{(\log (2 /(1-r)))^{\mu q}} d r\right)^{1 / q}+\left(\int_{|x|}^{1} r^{n-1} \frac{(1-r)^{(m-\lambda) q}}{(\log (2 /(1-r)))^{\mu q}} d r\right)^{1 / q} \\
& \quad=I(x)+J(x) .
\end{aligned}
$$

By elementary calculus, we obtain that for $|x| \in[1 / 2,1)$,

$$
\begin{aligned}
I(x) & \sim \delta(x)\left(\int_{0}^{1 / 2} r^{n-1} d r+\int_{1 / 2}^{|x|}(1-r)^{(m-\lambda-1) q}\left(\log \left(\frac{2}{1-r}\right)\right)^{-\mu q} d r\right)^{1 / q} \\
& \sim \delta(x)\left[1+\left(\int_{4}^{2 /(1-|x|)} t^{(\lambda+1-m) q-2}(\log t)^{-\mu q} d r\right)^{1 / q}\right]
\end{aligned}
$$

Hence, we distinguish the following cases.

(i) If $\lambda>m-1 / p$, then we have

$$
I(x) \sim(\delta(x))^{m-\lambda+1 / q}\left(\log \frac{2}{\delta(x)}\right)^{-\mu} .
$$

(ii) If $\lambda<m-1 / p$, then we have

$$
I(x) \sim \delta(x) .
$$


10 Existence result for a nonlinear polyharmonic problem

(iii) If $\lambda=m-1 / p$, then we have

$$
I(x) \sim \begin{cases}\delta(x)\left(\log \log \frac{2}{1-|x|}\right)^{1 / q} & \text { if } \mu=\frac{1}{q}, \\ \delta(x)\left(\log \frac{2}{1-|x|}\right)^{1 / q-\mu} & \text { if } \mu<\frac{1}{q} \\ \delta(x) & \text { if } \mu>\frac{1}{q}\end{cases}
$$

On the other hand, we have

$$
J(x) \sim\left(\int_{2 /(1-|x|)}^{\infty} t^{(\lambda-m) q-2}(\log t)^{-\mu q}\right)^{1 / q} .
$$

So, by elementary calculus, we obtain

$$
J(x) \sim \begin{cases}(\delta(x))^{m-\lambda+1 / q}\left(\log \frac{2}{\delta(x)}\right)^{-\mu} & \text { if } \lambda<m+1-\frac{1}{p}, \mu \in \mathbb{R}, \\ \left(\log \frac{2}{\delta(x)}\right)^{1 / q-\mu} & \text { if } \lambda=m+1-\frac{1}{p}, \mu>\frac{1}{q} .\end{cases}
$$

Hence, for $|x| \in[1 / 2,1)$, we have

$$
\begin{aligned}
& \left(\int_{0}^{1} r^{n-1} \frac{(1-r)^{(m-\lambda-1) q}}{(\log (2 /(1-r)))^{\mu q}}(\rho(|x| \vee r))^{q} d r\right)^{1 / q} \\
& \qquad \begin{cases}(\delta(x))^{m-\lambda+1 / q}\left(\log \frac{2}{\delta(x)}\right)^{-\mu} & \text { if } m-\frac{1}{p}<\lambda<m+1-\frac{1}{p}, \mu \in \mathbb{R}, \\
\delta(x)\left(\log \frac{2}{\delta(x)}\right)^{1 / q-\mu} & \text { if } \lambda=m-\frac{1}{p}, \mu<\frac{1}{q} \\
\delta(x)\left(\log \log \frac{2}{\delta(x)}\right)^{1 / q} & \text { if } \lambda=m-\frac{1}{p}, \mu=\frac{1}{q} \\
\delta(x) & \text { if } \lambda=m-\frac{1}{p}, \mu>\frac{1}{q} \\
\delta(x) & \text { if } \lambda<m-\frac{1}{p}, \mu \in \mathbb{R} \\
\left(\log \frac{2}{\delta(x)}\right)^{1 / q-\mu} & \lambda=m+1-\frac{1}{p}, \mu>\frac{1}{q} .\end{cases}
\end{aligned}
$$

Thus, by combination of the two cases, we obtain the result. 


\section{Proof of the main existence result}

In this section, we aim at proving Theorem 1.2, so we need the following result (see [10, Proposition 4]).

Proposition 3.1. For a fixed nonnegative function $q$ in $K_{m, n}$,

$$
M_{q}:=\left\{f \in \mathscr{B}^{+}(B) ; f \leq q\right\} \text {, }
$$

then the family of functions

$$
\left\{\frac{1}{(\delta(x))^{m-1}} \int_{B}(\delta(y))^{m-1} G_{m, n}(x, y) f(y) d y ; f \in M_{q}\right\}
$$

is relatively compact in $C_{0}(B)$.

Proof of Theorem 1.2. Assuming $\mathrm{H}_{1}-\mathrm{H}_{3}$, we will use a fixed point argument to construct a solution of the problem $(P)$.

By (1.6), the function $\theta(x)=q(x) /(\delta(x))^{m-1} \in K_{m, n}$, then using Proposition 3.1, we obtain

$$
\left\|V\left((\delta(\cdot))^{m-1} \theta\right)\right\|_{\infty}=\|V q\|_{\infty}<\infty .
$$

Consequently, we deduce by (1.7) and (1.8), that $p$ is a nontrivial nonnegative function in $L_{\text {loc }}^{1}(B)$, then there exists a compact $\mathrm{K}$ in $B$ such that we have

$$
0<\alpha:=\int_{K}(\delta(y))^{m} p(y) d y<\infty .
$$

Put $\beta:=\min \left\{(\delta(x))^{m} ; x \in K\right\}$, then from (1.8), we deduce that there exists a constant $a>0$ such that

$$
c \alpha f(a \beta) \geq a \text {. }
$$

Take $0<\eta<1 /\|V q\|_{\infty}$, then we deduce by (1.9) that there exists $\rho>0$ such that for $t \geq \rho$, we have $g(t) \leq \eta t$. Hence, put $\gamma=\sup _{0 \leq t \leq \rho} g(t)$, then we obtain

$$
0 \leq g(t) \leq \eta t+\gamma \quad \forall t \geq 0
$$

Finally, it follows from (2.2) and (1.6) that there exists a constant $c_{1}>0$ such that for each $x \in B$,

$$
c_{1}(\delta(x))^{m} \leq V q(x)
$$

and using further the hypothesis $\left(\mathrm{H}_{2}\right)$ and the Proposition 3.1, we deduce that $\| V(\varphi(\cdot$, $\left.\left.a(\delta(\cdot))^{m}\right)\right) \|_{\infty}<\infty$. Hence, let $b=\max \left\{a / c_{1},\left(\eta\left\|V\left(\varphi\left(\cdot, a(\delta(\cdot))^{m}\right)\right)\right\|_{\infty}+\gamma\right) /\left(1-\eta\|V q\|_{\infty}\right)\right\}$ and consider the closed convex set

$$
\Lambda:=\left\{u \in C_{0}(B): a(\delta(\cdot))^{m} \leq u \leq V\left(\varphi\left(\cdot, a(\delta(\cdot))^{m}\right)\right)+b V q\right\} .
$$


Obviously, by (3.7) we have that the set $\Lambda$ is nonempty. Define the integral operator $T$ on $\Lambda$ by

$$
T u(x):=\int_{B} G_{m, n}(x, y)[\varphi(y, u(y))+\psi(y, u(y))] d y \quad \forall x \in B .
$$

Let us prove that $T \Lambda \subset \Lambda$. Let $u \in \Lambda$ and $x \in B$, then by (1.7) and (3.6) we have

$$
\begin{aligned}
T u(x) \leq & V\left(\varphi\left(\cdot, a(\delta(\cdot))^{m}\right)\right)(x)+\int_{B} G_{m, n}(x, y) q(y) g(u(y)) d y \\
\leq & V\left(\varphi\left(\cdot, a(\delta(\cdot))^{m}\right)\right)(x)+\int_{B} G_{m, n}(x, y) q(y)[\eta u(y)+\gamma] d y \\
\leq & V\left(\varphi\left(\cdot, a(\delta(\cdot))^{m}\right)\right)(x) \\
& +\int_{B} G_{m, n}(x, y) q(y)\left[\eta\left(\left\|V\left(\varphi\left(\cdot, a(\delta(\cdot))^{m}\right)\right)\right\|_{\infty}+b\|V q\|_{\infty}\right)+\gamma\right] d y .
\end{aligned}
$$

Since $\eta\left(\left\|V\left(\varphi\left(\cdot, a(\delta(\cdot))^{m}\right)\right)\right\|_{\infty}\right)+\gamma \leq b\left(1-\eta\|V q\|_{\infty}\right)$, then

$$
\begin{aligned}
& \int_{B} G_{m, n}(x, y) q(y)\left[\eta\left(\left\|V\left(\varphi\left(\cdot, a(\delta(\cdot))^{m}\right)\right)\right\|_{\infty}+b\|V q\|_{\infty}\right)+\gamma\right] d y \\
& \quad \leq b \int_{B} G_{m, n}(x, y) q(y) d y=b V q(x), \\
& T u(x) \leq V\left(\varphi\left(\cdot, a(\delta(\cdot))^{m}\right)\right)(x)+b V q(x) .
\end{aligned}
$$

Moreover, from the monotonicity of $f$ and using the relations (1.7), (2.2), and (3.5), there exists a constant $c>0$ such that for each $x \in B$, we have

$$
\begin{aligned}
T u(x) & \geq \int_{B} G_{m, n}(x, y) \psi(y, u(y)) d y \geq c(\delta(x))^{m} \int_{B}(\delta(y))^{m} p(y) f(u(y)) d y \\
& \geq c f(a \beta)\left(\int_{K}(\delta(y))^{m} p(y) d y\right)(\delta(x))^{m} \geq a(\delta(x))^{m} .
\end{aligned}
$$

On the other hand, we have that for each $u \in \Lambda, \varphi(\cdot, u) \leq \varphi\left(\cdot, a(\delta(\cdot))^{m}\right)$ and $\psi(\cdot, u) \leq$ $g(u) q \leq b q$, which implies that

$$
\varphi(\cdot, u)+\psi(\cdot, u) \in \mathcal{M}_{\left(\varphi\left(\cdot, a(\delta(\cdot))^{m}\right)+b q\right)} .
$$

So we deduce by $\left(\mathrm{H}_{2}\right),(1.6)$, and Proposition 3.1 that $T \Lambda$ is relatively compact in $C_{0}(B)$. In particular, for all $u \in \Lambda, T u \in C_{0}(B)$ and we deduce that $T \Lambda \subset \Lambda$.

Next, we prove the continuity of $T$ in $\Lambda$. Let $\left(u_{k}\right)_{k}$ be a sequence in $\Lambda$ which converges uniformly to a function $u$ in $\Lambda$. Then since $\varphi$ and $\psi$ are continuous with respect to the second variable, we deduce by the dominated convergence theorem that

$$
T u_{k}(x) \longrightarrow T u(x) \text { as } x \longrightarrow \infty \text {. }
$$

Now, since $T \Lambda$ is relatively compact in $C_{0}(B)$, then we obtain the uniform convergence. Hence $T$ is a compact operator mapping from $\Lambda$ to itself. So by the Schäuder fixed point 
theorem, there exists a function $u \in \Lambda$ such that

$$
u(x)=\int_{B} G_{m, n}(x, y)[\varphi(y, u(y))+\psi(y, u(y))] d y \quad \forall x \in B .
$$

Finally, we need to verify that $u$ is a positive continuous solution for the problem $(P)$. Indeed, since the functions $\theta(x)=q /(\delta(\cdot))^{m-1}$ and $x \mapsto \varphi\left(\cdot, a(\delta(\cdot x))^{m}\right) /(\delta(x \cdot))^{m-1}$ are in $K_{m, n}$, we deduce by (3.13) and Proposition 2.2 that the function

$$
\varphi(\cdot, u)+\psi(\cdot, u) \in L_{\text {loc }}^{1}(B)
$$

holds. Moreover, since $u \in C_{0}(B)$, we deduce from $(3.15)$ that $V(\varphi(\cdot, u)+\psi(\cdot, u))$ is in $L_{\text {loc }}^{1}(B)$. Hence $u$ satisfies (in the sense of distributions) the equation

$$
(-\Delta)^{m} u=\varphi(\cdot, u)+\psi(\cdot, u) \quad \text { in } B .
$$

Moreover, since $u$ satisfies

$$
a \delta(x) \leq \frac{u(x)}{(\delta(x))^{m-1}} \leq \frac{1}{(\delta(x))^{m-1}}\left(V\left(\varphi\left(\cdot, a \delta^{m}\right)\right)(x)+b V q(x)\right),
$$

then it follows from hypotheses $\left(\mathrm{H}_{2}\right),(1.6)$, and Proposition 3.1 that $\lim _{|x| \rightarrow 1}(u(x) /$ $\left.(\delta(x))^{m-1}\right)=0$. This completes the proof.

Example 3.2. Let $\alpha, \beta \geq 0$ such that $0 \leq \alpha+\beta<1, \max (\sigma, \lambda)<m$, and $\gamma>0$. Then the problem

$$
\begin{gathered}
(-\Delta)^{m} u=(\delta(x))^{m \gamma-\sigma}(u(x))^{-\gamma}+(\delta(x))^{-\lambda}(u(x))^{\alpha} \log \left(1+(u(x))^{\beta}\right) \quad \text { in } B, \\
u>0, \\
\lim _{|x| \rightarrow 1} \frac{u(x)}{(\delta(x))^{m-1}}=0
\end{gathered}
$$

has a positive solution $u \in C_{0}(B)$ satisfying

$$
a(\delta(x))^{m} \leq u(x) \leq b(\delta(x))^{m}
$$

where $a, b$ are positive constants.

\section{References}

[1] I. Bachar, H. Mâagli, S. Masmoudi, and M. Zribi, Estimates for the Green function and singular solutions for polyharmonic nonlinear equation, Abstract and Applied Analysis 2003 (2003), no. $12,715-741$.

[2] T. Boggio, Sulle funzioni di Green d'ordine $m$, Rendiconti del Circolo Matematico di Palermo 20 (1905), 97-135.

[3] M. G. Crandall, P. H. Rabinowitz, and L. Tartar, On a Dirichlet problem with a singular nonlinearity, Communications in Partial Differential Equations 2 (1977), no. 2, 193-222.

[4] J. I. Diaz, J.-M. Morel, and L. Oswald, An elliptic equation with singular nonlinearity, Communications in Partial Differential Equations 12 (1987), no. 12, 1333-1344. 


\section{Existence result for a nonlinear polyharmonic problem}

[5] A. L. Edelson, Entire solutions of singular elliptic equations, Journal of Mathematical Analysis and Applications 139 (1989), no. 2, 523-532.

[6] P. R. Garabedian, A partial differential equation arising in conformal mapping, Pacific Journal of Mathematics 1 (1951), no. 4, 485-524.

[7] T. Kusano and C. A. Swanson, Entire positive solutions of singular semilinear elliptic equations, Japanese Journal of Mathematics. New Series 11 (1985), no. 1, 145-155.

[8] A. V. Lair and A. W. Wood, Large solutions of sublinear elliptic equations, Nonlinear Analysis. Theory, Methods \& Applications 39 (2000), no. 6, 745-753.

[9] A. C. Lazer and P. J. McKenna, On a singular nonlinear elliptic boundary-value problem, Proceedings of the American Mathematical Society 111 (1991), no. 3, 721-730.

[10] H. Mâagli, F. Toumi, and M. Zribi, Existence of positive solutions for some polyharmonic nonlinear boundary-value problems, Electronic Journal of Differential Equations 2003 (2003), no. 58, 119.

[11] J.-P. Shi and M. Yao, On a singular nonlinear semilinear elliptic problem, Proceedings of the Royal Society of Edinburgh. Section A. Mathematics 128 (1998), no. 6, 1389-1401.

Sonia Ben Othman: Département de Mathematiques, Faculté des Sciences de Tunis, Campus Universitaire, 1060 Tunis, Tunisia

E-mail address: sonia.benothman@fsb.rnu.tn 


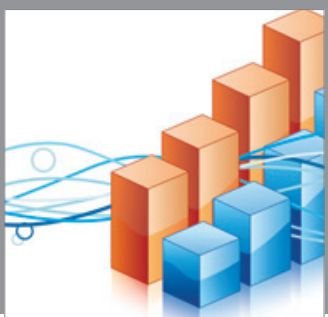

Advances in

Operations Research

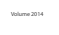

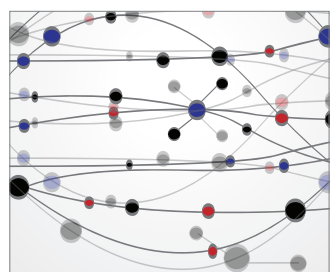

\section{The Scientific} World Journal
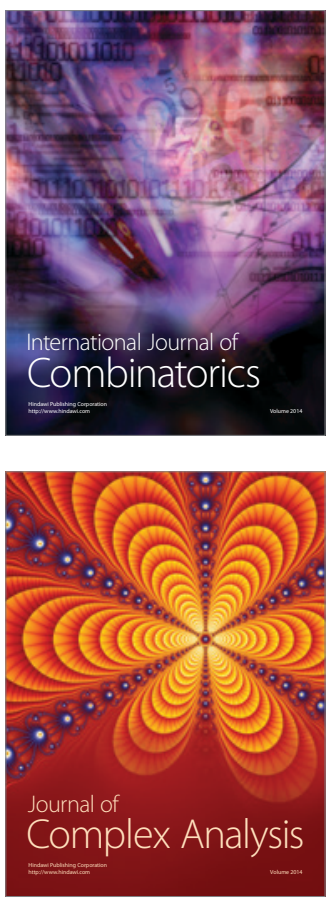

International Journal of

Mathematics and

Mathematical

Sciences
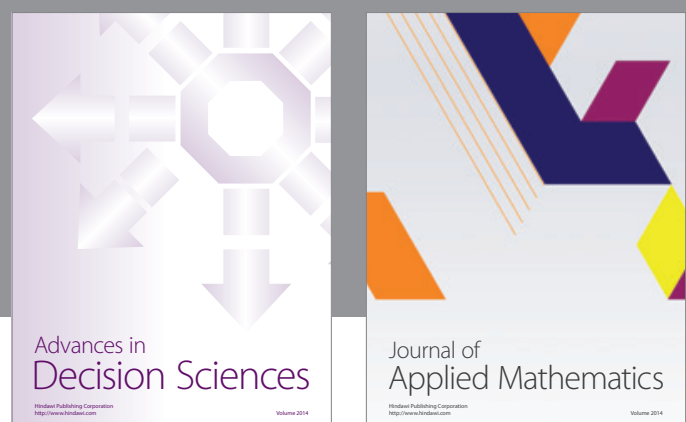

Journal of

Applied Mathematics
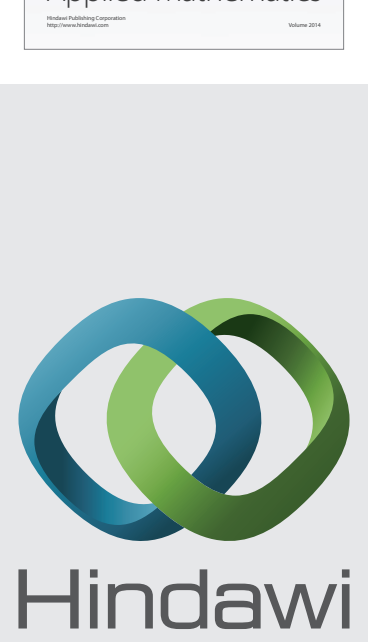

Submit your manuscripts at http://www.hindawi.com
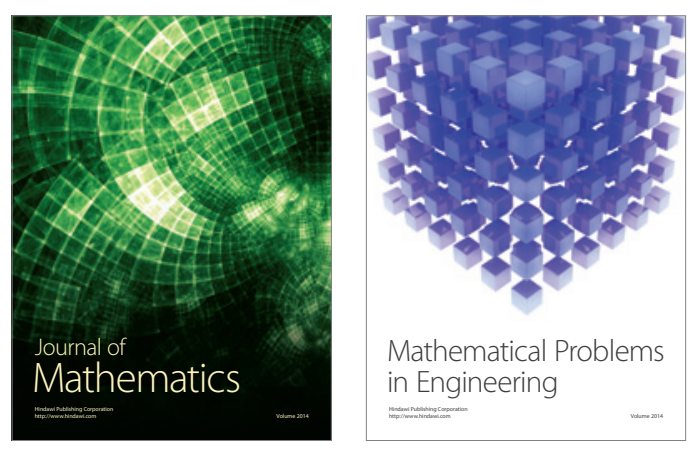

Mathematical Problems in Engineering
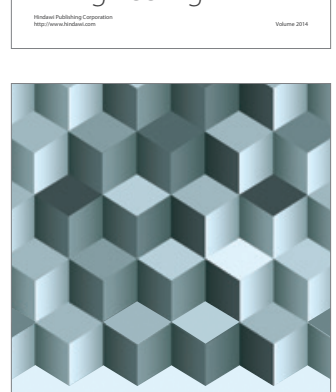

Journal of

Function Spaces
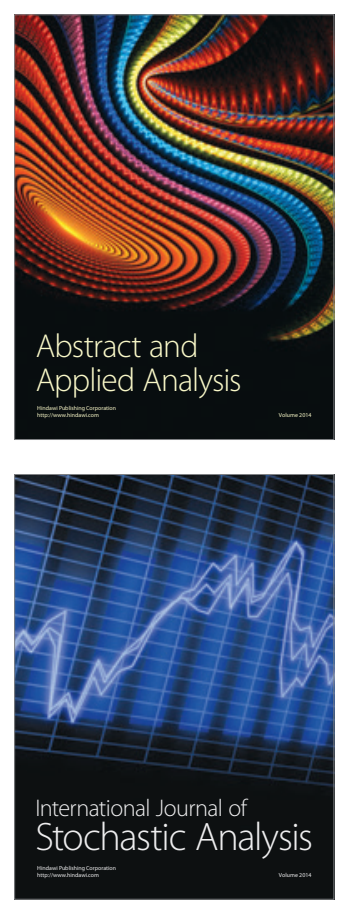

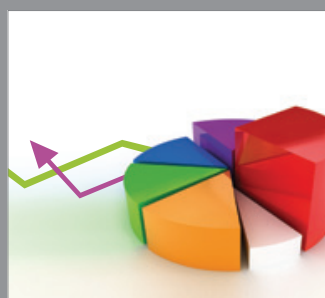

ournal of

Probability and Statistics

Promensencen
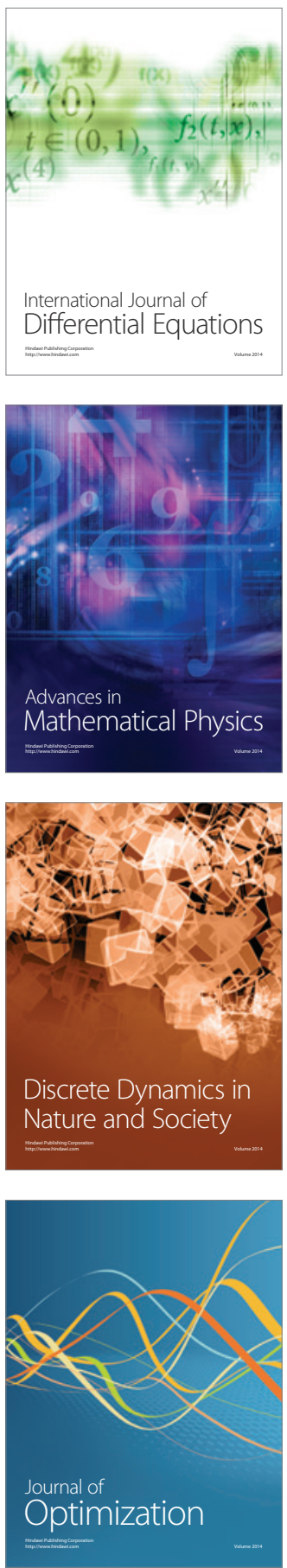